\title{
A Drosophila toolkit for defining gene function in spermatogenesis
}

\author{
N A Siddall and G R Hime \\ Department of Anatomy and Neuroscience, The University of Melbourne, Parkville, Victoria, Australia \\ Correspondence should be addressed to G Hime; Email: g.hime@unimelb.edu.au
}

\begin{abstract}
Expression profiling and genomic sequencing methods enable the accumulation of vast quantities of data that relate to the expression of genes during the maturation of male germ cells from primordial germ cells to spermatozoa and potential mutations that underlie male infertility. However, the determination of gene function in specific aspects of spermatogenesis or linking abnormal gene function with infertility remain rate limiting, as even in an era of CRISPR analysis of gene function in mammalian models, this still requires considerable resources and time. Comparative developmental biology studies have shown the remarkable conservation of spermatogenic developmental processes from insects to vertebrates and provide an avenue of rapid assessment of gene function to inform the potential roles of specific genes in rodent and human spermatogenesis. The vinegar fly, Drosophila melanogaster, has been used as a model organism for developmental genetic studies for over one hundred years, and research with this organism produced seminal findings such as the association of genes with chromosomes, the chromosomal basis for sexual identity, the mutagenic properties of $\mathrm{X}$-irradiation and the isolation of the first tumour suppressor mutations. Drosophila researchers have developed an impressive array of sophisticated genetic techniques for analysis of gene function and genetic interactions. This review focuses on how these techniques can be utilised to study spermatogenesis in an organism with a generation time of 9 days and the capacity to introduce multiple mutant alleles into an individual organism in a relatively short time frame.

Reproduction (2017) 153 R121-R132
\end{abstract}

\section{Introduction}

Infertility in males can be caused by many different factors including genetic mutations that affect the spermatogenic process. It has been estimated that roughly 2000 genes are involved in the processes of human spermatogenesis, from testicular development through to the end stages of spermiogenesis (Bhasin et al. 2000). Identification of the genes involved in mammalian spermatogenesis has largely occurred through the study of mouse models (Yatsenko et al. 2010) as (apart from pituitary and endocrine disorders or aberrations associated with the Y-chromosome) most cases of human male infertility are idiopathic and any genes identified are difficult to functionally verify in humans (Waclawska \& Kurpisz 2012). Although mouse models remain significantly important for the identification and functional characterisation of genes involved in spermatogenesis, other studies have also highlighted the powerful use of Drosophila melanogaster genetics to uncover genes involved in conserved spermatogenic processes. A recent study by $\mathrm{Wu}$ and coworkers, for example, perfectly demonstrates the potential of Drosophila for discovery and analysis of genes that are required for male fertility. In their well-designed study, $\mathrm{Wu}$ and coworkers utilised a multi-faceted approach in
Drosophila to demonstrate a conserved requirement for the major spliceosome in spermatogenesis $(\mathrm{Wu}$ et al. 2016). The authors achieved this by identifying single nucleotide polymorphisms in humans that associate with non-obstructive azoospermia (NOA) and analysing the corresponding candidate loci in Drosophila using a range of tools that will be described in this review, thus demonstrating the advantages of analysing genes of interest to uncover function in Drosophila. The purpose of this review is not to compare human or mammalian spermatogenesis with Drosophila spermatogenesis per se, but to highlight the powerful technologies and resources available in Drosophila melanogaster that can be taken advantage of to rapidly uncover gene function, and how this can be useful for researchers studying spermatogenesis in other systems.

\section{Drosophila anatomy}

The genetic tractability of Drosophila melanogaster, along with its incredibly fast (less than 2 weeks) generation cycle has made it an excellent model system to discover and study genes and signalling pathways required for both early and late spermatogenesis. Spermatogenesis in Drosophila adults occurs in a coiled, blind-ended 
tube (Fig. 1A). Approximately, 8-12 germline stem cells (GSCs) reside at the apical tip of this tube, and generally divide asymmetrically to produce two cells: a daughter stem cell and a gonialblast (GB) (Fig. 1B and C). In a very similar process to mammalian spermatogenesis, the $\mathrm{GB}$ undergoes four rounds of mitotic divisions to generate a cyst of 16 inter-connected spermatogonial cells that ultimately differentiate into mature spermatocytes. These spermatocytes initiate a meiotic program that results in the generation of 64 haploid spermatids (Fuller 1993). After maturation and elongation, sperm are transported to the seminal vesicle (Fuller 1993). The developing germline cells are enclosed by somatic cyst cells, which are derived from cyst stem cells (CySCs), with two CySCs surrounding each GSC (Fig. 1B and C). Drosophila spermatogenesis has been suggested to be analogous to the mouse neonate as the CySCs maintain their proliferative potential to continuously generate nascent Sertoli-like cyst cells. In the Drosophila testis, there is a high degree of cross-talk between the soma and germline, with the two populations of cells being highly inter-dependent in order for spermiogenesis to occur (Zoller \& Schulz 2012). The similarity in the tight association between germline and soma in Drosophila and mammalian testes implies that signalling pathways between germline and soma may be conserved from flies to mammals.

\section{Tools to study the Drosophila testis niche: an imaging approach}

GSCs reside in what is referred to as a stem cell niche (Li \& Xie 2005), which comprises a cluster of 10-15 non-dividing somatic hub cells, somatic cyst stem cells (CySCs) and the GSCs themselves (Fig. 1C). GSC homeostasis is dependent upon cross-talk of signalling pathways between somatic CySCs and hub cells with GSCs (reviewed in de Cuevas \& Matunis 2011, Greenspan et al. 2015). Janus Kinase-Signal Transducer and Activator of Transcription (JAK-STAT) signalling (Kiger et al. 2001, Tulina \& Matunis 2001, Leatherman \& Dinardo 2010); the bone morphogenesis protein (BMP) pathway (Kawase et al. 2004, Michel et al. 2011, Inaba et al. 2015) and hedgehog signalling (Michel et al. 2012, Amoyel et al. 2013, Zhang et al. 2013) are all involved in regulating early germ cell development, with short-range signals being received in the GSCs from either (or both) the hub cells or CyCSs.

The Drosophila testis niche has been extensively studied, and one of the advantages of using Drosophila to understand the genetic complexities of niches is that studies can easily be carried out in vivo (de Cuevas \& Matunis 2011). Cells in the apical tip of the testis are well defined anatomically and can be visually identified using fluorescent microscopy techniques and antibodies, which are readily available
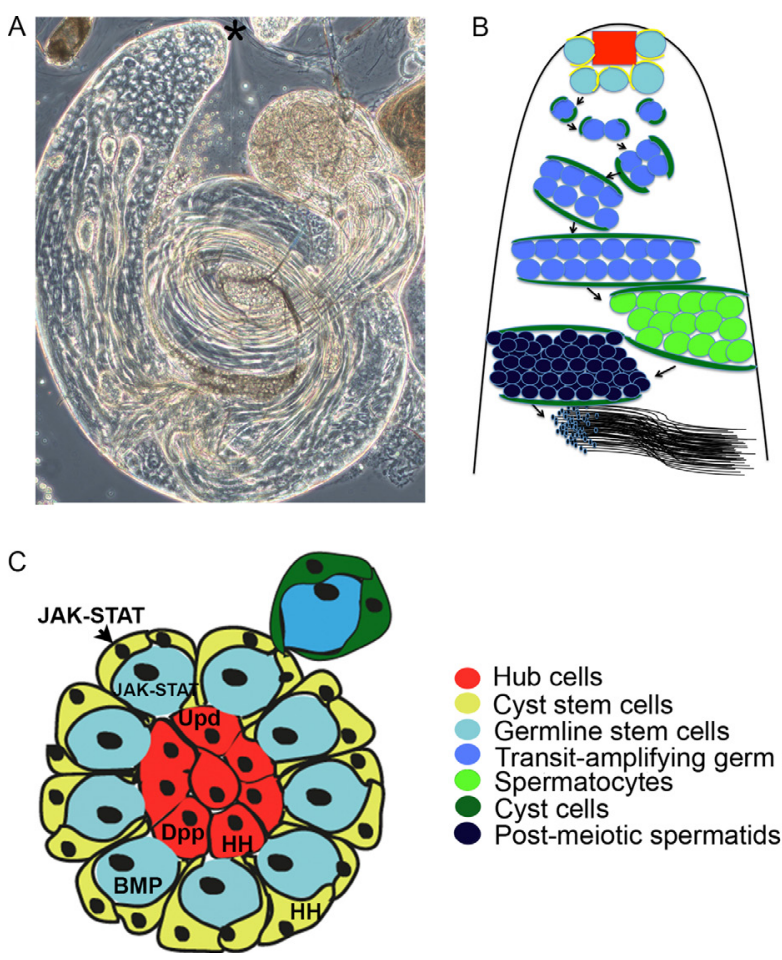

Hub cells

Cyst stem cells

Germline stem cells

Transit-amplifying germ cells

Spermatocytes

Cyst cells

Post-meiotic spermatids

Figure 1 (A) Phase-contrast image of a wild-type testis. All stages of spermatogenesis can be observed in a single testis after dissection, light squashing and mounting and visualization using phase-contrast microscopy. Early germ cells reside at the testis apex, marked by an asterisk. Motile sperm can also be visualized with this technique as sperm is released from the seminal vesicle at the testis base.

(B) Cartoon schematic diagram of spermatogenesis. Somatic hub cells (red) reside at the testis apex and act as a niche supporting 8-10 germline stem cells (represented in light blue). Cyst progenitor cells (yellow) surround each germline stem cell and also provide signals to germline stem cells to ensure proper differentiation and division. GSCs divide to produce daughter gonialblasts (blue), which are surrounded by differentiated somatic cyst cells (green). The gonialblast undergoes 4 rounds of division, which is the transit-amplifying stage of germ cell development (blue). A cyst of 16 spermatogonial cells undergo a growth phase and differentiate in mature spermatocytes (light green). Spermatocytes undergo meiosis to produce 64 haploid round spermatids (dark blue), which differentiate into 64 individualized sperm. (C) A cartoon schematic of the cells and local signalling pathways activated within the testis niche. Somatic hub cells (red) secrete the ligand unpaired (Upd), which in turn activate Janus Kinase-Signal Transducer and Activator of Transcription (JAK-STAT) signalling in adjacent GSCs (light blue) and CySCs (yellow) (Leatherman \& DiNardo 2008, Flaherty et al. 2010). Hedgehog (HH) signals are also received by the CySCs from the hub cells (Amoyel et al. 2013, Zhang et al. 2013). The ligand Decapentaplegic (Dpp) is also secreted from the hub cells to activate Bone morphogenetic protein (BMP) signalling in adjacent GSCs (Inaba et al. 2015) GSCs divide to self-renew and produce a daughter gonialblast (blue). CySCs (yellow) similarly divide to self-renew and produce daughter cyst cells (green), which are fully differentiated and no longer divide, but rather encapsulate and support dividing cysts of germ cells.

to the Drosophila community (Fig. 2A, B, C and D). Many of these antibodies can be publically sourced from the Developmental Studies Hybridoma Bank (DSHB, http://dshb.biology.uiowa.edu/) at the University 

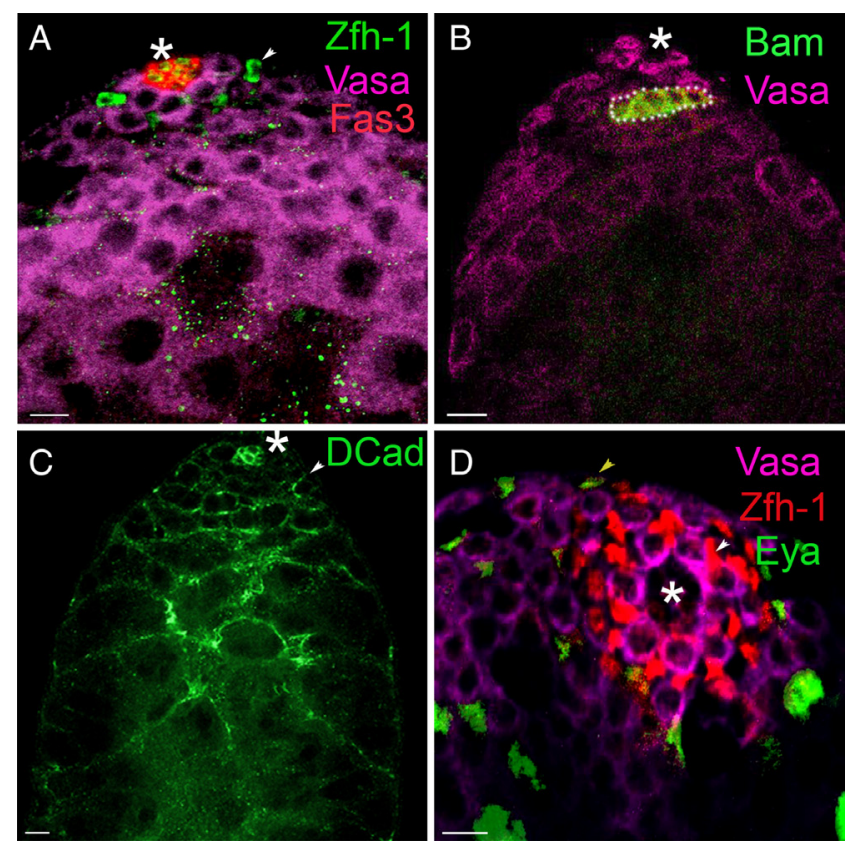

Figure 2 (A, B, C and D) Adult wild-type testes labelled with cell-specific antibodies. The asterisk in each image denotes the hub. Scale bars represent $10 \mu \mathrm{m}$. (A) Fasciclin-3 (red) labels the hub at the very apex of the testis. Vasa (magenta) labels germ cells, including GSCs surrounding the hub and differentiating germ cells throughout the testis. Zfh1 (green) labels the somatic hub and cyst stem cells directly adjacent to the hub (white arrow), but expression is lost upon differentiation of CySCs to cyst cells. (B) Bag of Marbles (Bam, green) is a useful antibody to detect differentiated early germ cells as it labels cells in the 4-16 stage of spermatogenesis. A cyst of 4 spermatogonial cells (dotted outline) is depicted in this testis, with all germ cells labelled with Vasa (magenta). (C) An antibody against $\mathrm{DE}$-cadherin (green) is useful to mark the hub and outline spermatogonial cysts. (D) Zfh1 expression (red) is useful for labelling undifferentiated cyst cells, whereas Eyes-Absent (Eya, green) labels differentiated cyst cells.

of lowa. Some of the more commonly used antibodies available from DSHB to label hub cells include antiFasciclin 3 (Gonczy et al. 1992), anti-Armadillo and anti-dE-cadherin (Yamashita et al. 2003) or anti-dNcadherin (Siddall et al. 2006). Antibodies that detect DE-cadherin and Armadillo expression are also useful for defining developing germline cysts in the apex of the testis. Antibodies that detect Vasa protein expression, which labels germ cells in the testis from stem cells to spermatocytes (Fig. 2) (Lasko \& Ashburner 1988), are available from both DSHB and Santa Cruz Biotechnology (http://www.scbt.com/). Antibodies directed against the Bag-of-Marbles (Bam) protein label germ cells at the 4-16 cell stage of spermatogonial development (McKearin \& Ohlstein 1995), Anti-Eyes Absent (Eya), available from DSHB, labels differentiated cyst cells (Fabrizio et al. 2003) but is notably absent from CySCs (Leatherman \& Dinardo 2008). Anti-Broad-core (DSHB) can be used to label CySCs and progeny (Siddall et al. 2006). Anti-1B1 (adducin-related protein) and anti- $\alpha$-Spectrin (both from DSHB) label the fusome connecting germ cells (Lin et al. 1994). Other common antibodies used for testis niche analysis include anti-Zfh1 and anti-Traffic-Jam (Li etal.2003, Leatherman \& Dinardo 2008) for labelling CySC and anti-Stat92E for labelling of GSCs and CySCs (Flaherty et al. 2010). In addition to antibodies, many fly reporter lines are available for the use of visualization of LacZ (detectable by X-gal staining or with an anti- $\beta$-galactosidase antibody), or fluorescent reporters, such as green fluorescent protein (GFP), in specific cells. Such lines include neuralized-lacZ and fringe-lacZ for marking early germ cells and cyst cells respectively (Terry et al. 2006), bag-of-marbles-GFP for labelling transit-amplifying gonial cells from the 4 to 16-cell stage (Chen \& McKearin 2003) and vasa-GFP for labelling of germ cells (Kelso et al. 2004) amongst many others. These fly lines can be sourced from various stock centres, all of which are listed on Flybase (http:// flybase.org/), a database used daily by the Drosophila community to glean information about genes of interest.

Recent development of transposon-based technologies has led to the generation of new tools to create GFPtagged lines for the visualization of protein and gene expression in different tissues. In Drosophila, the MinosMediated Integration Cassette (MiMIC) is a specialized transposon that carries two inverted attP sites that allow conversion of loci through $\phi C 31$ recombinase-mediated cassette exchange (RMCE) (Venken et al. 2011). These transposons contain a dominant body colour marker for easy screening and a stop cassette with a splice acceptor. Because of the stop cassette, when this transposon lands in an intron in the correct orientation, the result is a gene mutation. The presence of the attP sites allow for cassette exchange by simple recombination methods, thus allowing for the conversion of a MiMIC insertion into a protein-trap-containing GFP if the insertion is in a coding intron or a gene-trap if the MiMIC insertion is in the $5^{\prime}$ noncoding intron (Venken et al. 2011, Gnerer et al. 2015, Nagarkar-Jaiswal et al. 2015a). Over 7400 MiMIC insertions have been generated (Nagarkar-Jaiswal et al. 2015a), and most are publicly available from the Bloomington Drosophila Stock Center (www.flystocks. bio.indiana.edu). Although this technology has not been used to study the expression of spermatogenesis genes in Drosophila, it enables the potential for collaboration between mammalian and fly researchers to conduct such studies. For example, we have tabulated a list of 60 MiMIC lines that have been recombineered to convert the insertion into a protein-trap or gene-trap tagged with GFP and noted the human orthologue (Table 1). The GFP tag would allow high-resolution analysis of expression of potential interesting genes prior to conducting any further mutational studies.

In Drosophila, the use of antibodies and reporter lines coupled with imaging techniques has provided remarkable insights into mechanisms that control early germ cell behaviour. For microscopy techniques, both 
Table 1 This table lists sixty genes (Nagarkar-Jaiswal et al. 2015a) where the MiMIC transposable element has been recombineered to convert the insertion into a protein-trap or gene-trap tagged with GFP. Fifty of the tagged Drosophila genes have direct human orthologues, eight have related human genes (asterisk) and two appear to not have related genes in the human genome. Most of these genes are yet to be examined for functions in spermatogenesis.

\begin{tabular}{|c|c|c|}
\hline MiMIC line & Fly gene & Human gene \\
\hline MI00957 & GlyP & PYGM \\
\hline MI00963 & CG42732 & KCNT1 \\
\hline MI00996 & CG17349 & PACRGL \\
\hline MI01169 & CG10086 & $A R R D C 2 *$ \\
\hline MI01190 & CG32413 & $Q P C T^{*}$ \\
\hline MI01427 & RhoGEF3 & ARHGEF9 \\
\hline MI01568 & stops & $A S B 17$ \\
\hline MI01568 & Gycbeta100B & GUCY1B3 \\
\hline MI01574 & CG34384 & $D G K H$ \\
\hline MI01790 & MTA1-like & MTA3 \\
\hline MI01874 & eys & EYS \\
\hline MI02112 & CG5830 & CTDSP1 \\
\hline MI02221 & CG10426 & INPP5E \\
\hline MI02467 & $s f l$ & NDST2 \\
\hline MI02765 & loh & THSD4 \\
\hline MI02777 & CG6356 & SLC22A14 \\
\hline MI03218 & CG5535 & SLC7A1 \\
\hline MI03390 & CG7139 & $N 4 B P 2$ \\
\hline MI03727 & NKAIN & NKAIN3 \\
\hline MI03836 & $s c b$ & ITGA4 \\
\hline MI03838 & DIP-zeta & NTM $^{*}$ \\
\hline MI03943 & zip & MYH1O \\
\hline MI04094 & CG11576 & SLC52A3 \\
\hline MI04101 & Rgk3 & $R R A D^{*}$ \\
\hline MI04721 & CG34371 & SICLEC5* \\
\hline MI04868 & $D /$ & DLL1 \\
\hline MI05333 & kay & FOS \\
\hline MI05804 & $C C A P-R$ & NPSR 1 \\
\hline MI06288 & Alh & MLLT6 \\
\hline MI06507 & Cad86C & CDHR1 \\
\hline MI07766 & Nedd4 & NEDD4L \\
\hline MI07890 & CG33510 & No related gene \\
\hline MI07916 & Men-b & ME3 \\
\hline MI07934 & $M i$ & No related gene \\
\hline MI08468 & CG42324 & TJAP1 \\
\hline MI08481 & $k d m 2$ & $K D M 2 A$ \\
\hline MI08520 & PAPLA1 & DDHD2 \\
\hline MI08626 & Lip1 & PPFIA2* \\
\hline MI08698 & Sam-S & MAT2A \\
\hline MI08774 & form 3 & INF2 \\
\hline MI09216 & tws & $P P P 2 R 2 D$ \\
\hline MI09447 & Pde1c & PDE1A \\
\hline MI09888 & CG9541 & AK5 \\
\hline MI09912 & Tsp42Ee & CD63* \\
\hline MI10126 & CG13248 & $S L C 7 A 4$ \\
\hline MI10477 & CусA & CCNA2 \\
\hline MI10594 & Hrb98DE & HHRNPA2B1 \\
\hline MI10698 & a & PDZD2 \\
\hline MI11015 & Sap-r & PSAP \\
\hline MI11080 & Vha44 & ATP6V1C1 \\
\hline MI11086 & CG14085 & KIAA0825 \\
\hline MI11275 & mfas & TGFBI \\
\hline MI11796 & Nmdar1 & GRIN1 \\
\hline MI12194 & CG3764 & FNIP2 \\
\hline MI07575 & $\mid(2) g l$ & LLGL1 \\
\hline MI07659 & $d r p r$ & MEGF11 \\
\hline MI06587 & G $\alpha O$ & GNAO1 \\
\hline MI06777 & CG42321 & ATP8A $1^{*}$ \\
\hline MI07114 & CG5656 & $A L P P$ \\
\hline MI07336 & twin & CNOT6L \\
\hline
\end{tabular}

fixed tissue and live cell imaging protocols have been developed to help understand how Drosophila testis germline homeostasis is normally maintained (Sheng \& Matunis 2011, Sitaram et al. 2014). Sheng and Matunis used live cell imaging to show that GSCs can divide both symmetrically and asymmetrically in normal tissue. They further showed that in nutrient-deprived tissue, de-differentiation of spermatogonial cells into GSCs is a mechanism for niche regeneration (Sheng \& Matunis 2011). Inaba and coworkers used both fixedtissue and live cell imaging to describe the presence of GSC protrusions (nanotubes) that extend into the hub (Inaba et al. 2015). These nanotubes were visualized with a GFP- $\alpha 1$-tubulin84B reporter line, and live cell imaging was able to capture that these GSC nanotubes selectively traffic components of the niche signalling pathways between GSCs and hub cells. Their study was the first to demonstrate how short-range signals can be received by GSCs, and how GSCs may be distinguishable from their direct progeny (Inaba et al. 2015). The powerful imaging techniques coupled with the ever-increasing numbers of reporter lines, MiMICs and antibodies available give Drosophila researchers the ability to characterise the expression pattern of genes of unknown function or orthologues of mammalian genes known to be required for male fertility.

\section{Using GAL4-UAS and similar systems to study spermatogenesis}

Somatic and germ cells in the testis can be genetically manipulated using many tools available to Drosophila melanogaster researchers. One tool is the ability to express or knockdown genes in specific cells using the well-established GAL4-UAS system from yeast (Brand \& Perrimon 1993). In this system, the yeast transcription factor (GAL4) is expressed in a spatially restricted manner using a cell-/tissue-specific promoter. The GAL4 protein then activates the expression of transgenes under the control of an upstream activator sequence (UAS) to which the GAL4 protein can bind. This technique can be further enhanced by introducing a temperaturesensitive GAL80 (GAL80 $\left.{ }^{\text {ts }}\right)$ into the system, which inhibits GAL4 activation at permissive temperatures, but allows the expression of the UAS gene construct at restrictive temperatures, thus enabling stage and cellspecific expression of the gene of interest (reviewed in McGuire et al. 2004). Multiple GAL4 'driver' lines exist for the testis and enable the expression of transgenes in hub cells, in cyst stem cells, in differentiated cyst cells and in the early germline of the testis (Table 2) (reviewed in White-Cooper 2012, Demarco et al. 2014). Figure 3 shows an example of utilisation of the GAL4-UAS system to overexpress components of the BMP signalling pathway specifically in germ cells in the apex of the 
Table 2 Commonly used Gal4 driver lines in the Drosophila testis.

\begin{tabular}{llll}
\hline Cell type & Name of construct & Expression pattern - adult & Reference \\
\hline Germline & nanos-Gal4-VP16 & GSCs, spermatogonial & Van Doren et al. (1998) \\
& bam-Gal4-VP16 & C-16 stage of spermatogonial & Chen and McKearin (2003) \\
vasa-Gal4 & Germ cells & Zhao et al. (2013) \\
Soma - hub & Hub cells & Bras and Van Doren (2006) \\
Soma - hub and cyst cells & tj-Gal4 & Hub, CySCs, early cyst cells & Li et al. (2003) \\
& c833-Gal4 & Hub, CySCs, early cyst cells & Papagiannouli and Mechler (2009) \\
Soma - cyst cells & eya-Gal4 & Differentiated cyst cells & Leatherman and Dinardo (2008) \\
& ptc-Gal4 & CySCs and early cyst cells & Schulz et al. (2004) \\
& c729-Gal4 & Sheath cells, CySCs, early cyst cells & Papagiannouli and Mechler (2009) \\
& c587-Gal4 & CySCs and early cyst cells & Kawase et al. (2004) \\
& T155-Gal4 & Late cyst cells & Papagiannouli and Mechler (2009) \\
& Elfless-Gal4 & Tail cyst cells & Caldwell et al. (2008) \\
Germline and soma & Tub-Gal4 & Ubiquitous & Lee and Luo (1999) \\
\hline
\end{tabular}

testis. Expression of a constitutively active Thickveins (Tkv) transgene (UAS-Tkv ${ }^{\text {ACT }}$ ) in early germ cells with the nanos-GAL4 driver results in an over-proliferation of early germ cells, which can be readily visualized by phase-contrast microscopy (Fig. 3B) (Bunt \& Hime 2004). Tkv is a type I receptor for the decapentaplegic (Dpp) morphogen. In contrast, overexpression of a molecule that blocks Dpp activity, the inhibitory SMAD Daughters against Decapentaplegic (Dad), in the early germline with the nanos-GAL4 driver results in a loss of germ cells, which can also be readily visualized by phase-contrast microscopy (Fig. 3C). These experiments clearly highlight the power of the GAL4-UAS system in driving transgene expression in a cell-specific manner.

Our lab also recently conducted a screen and identified useful Gal4 driver lines for expression of transgenes in late spermatogonial cells and in early spermatocytes (Bunt et al. 2012), adding to the abundant collection of GAL4 driver lines available to researchers in Drosophila spermatogenesis. Table 2 lists some of the more commonly used GAL4 drivers to drive the expression of target UAS transgenes in various cell types in the testis.

The GAL4-UAS system can also be used to knockdown genes in specific cells by RNA interference (RNAi), where transgenic animals with an RNAi hairpin under the control of UAS have been generated for most genes in Drosophila and are available from the Vienna Drosophila Stock Center (VDRC, www.stockcenter. vdrc.at), from the Transgenic RNAi Project (TRiP) at the Bloomington Drosophila Stock Center and from the Japanese-based National Institute of Genetics (NIG). Using cell-specific GAL4 drivers to knockdown gene function by RNAi can enable cell-specific analysis of gene function.

Our lab has had continued success utilising the GAL4UAS-based strategy to study early spermatogenesis. For example, the RNA-binding protein Held Out Wings (HOW) was identified as a major player in the regulation of GSCs through an overexpression study, whereby overexpressing UAS-HOW specifically in early germ cells with the nanos-GAL4 driver caused a slight overproliferation of the early germline identified by phase- contrast microscopy (Monk et al. 2010). In this study, we also used transgenic RNAi lines and overexpression lines, together with other techniques, to demonstrate a requirement for $\mathrm{HOW}$ in maintaining GSCs and the numbers of spermatogonial cell divisions through regulation of Cyclin B and Bam (Monk et al. 2010).

Systems analogous to GAL4-UAS, such as the QF-QUAS binary system (Potter \& Luo 2011) and the LexA-lexAop system (Lai \& Lee 2006) have since been developed in Drosophila melanogaster and will become useful additional and complementary tools for the study of gene function in spermatogenesis (reviewed in del Valle Rodriguez et al. 2012), particularly for the ability to differentially regulate expression of separate genes within the one tissue. Regulation of genes within an intersecting pattern of promoter activities is now possible with the development of transgenic lines that drive half of a split-GAL4 molecule also. In this system, GAL4 activity is only achieved in cells where both halves of the functional molecule are expressed (Pfeiffer et al. 2010).

In addition to UAS-RNAi-based approaches to knockdown gene function, MiMIC technology also allows for knockdown in Drosophila, again, expanding the tools and resources available to Drosophila researchers. As described previously, insertion of a MiMIC in a coding intron in the correct orientation can disrupt gene function (Venken et al. 2011). Exchange of the MiMIC by RMCE with a specifically designed cassette causes the introduction of an artificial exon encoding a protein tag such as GFP. In most cases, this rescues gene function while simultaneously allowing for cellular detection of the protein encoded by the gene by GFP analysis (Nagarkar-Jaiswal et al. 2015b). These GFP-tagged proteins can be targeted for knockdown by making use of transgenic RNAi lines targeting GFP or by a ubiquitination-based deGradFP (degrade green fluorescent protein) approach thus allowing for a mechanism of protein knockdown without the potential complication of knocking down off targets (Nagarkar-Jaiswal et al. 2015b). deGRADFP is a method for inactivating GFP-tagged proteins. A single-domain antibody fragment against GFP (vhhGFP4) is fused to the 

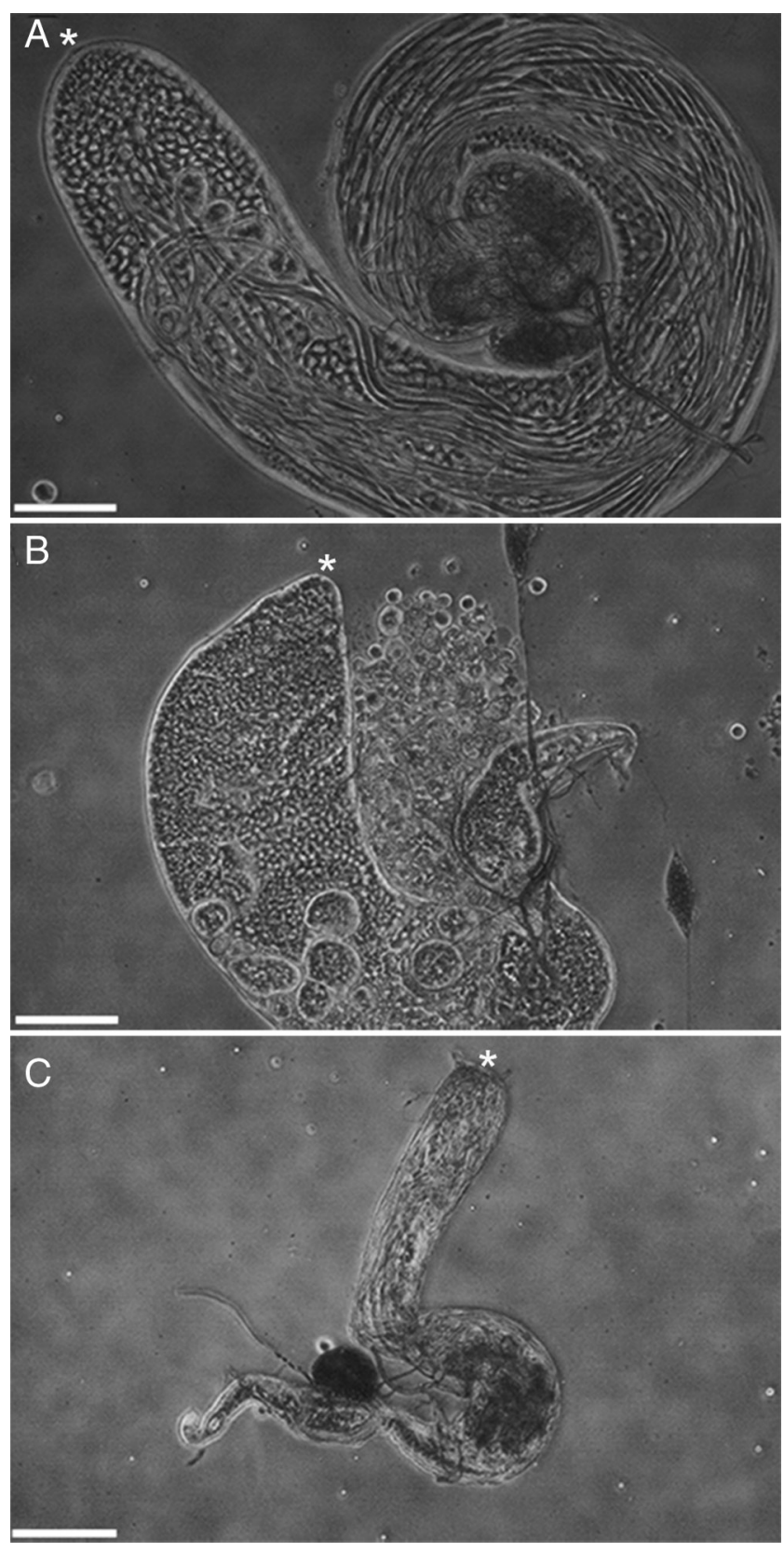

Figure 3 Phase-contrast imaging for analysis of the effects of Dpp signalling in the testis using the GAL4-UAS system. Scale bars represent $50 \mu \mathrm{m}$; asterisk denotes the testis apex. (A) Wild-type testis shows cells in all stages of development, from early germ cells at the apex through to differentiated sperm. (B) Phase-contrast image of a testis where nosGAL4 has been used to drive a UAS-Tkv ${ }^{\text {ACT }}$ construct in early germ cells. The testis is smaller and filled with undifferentiated small cells, and there is no evidence of sperm maturation. (C) Phase-contrast image of a testis where nosGAL4 has been used to drive a UAS-dad construct in early germ cells. The testis is smaller in size and has many less germ cells compared to a wild-type testis.

F-box domain from the SImb protein $\mathrm{N}$-terminus (NsImb) and expressed under UAS control. The fusion protein binds GFP and directs it to the proteasome for degradation. If gene function is provided by a GFP fusion construct, expression of the NsImb-vhhGFP4 fusion protein results in loss-of-function phenotypes in GAL4expressing cells (Caussinus et al. 2011). A list of MiMIC protein trap lines that is generated already is available at the Flypush website http://flypush.imgen.bcm.tmc.edu/ pscreen/rmce/.

\section{The use of FRT-mediated recombination, MARCM and other lineage-tracing techniques to study early spermatogenesis}

Although RNAi-based strategies have been useful in studying knockdown of genes in Drosophila in vivo, there are disadvantages to using this technology, such as variability in gene silencing or the potential of false positives (mainly from off-target effects) and false negatives (reviewed in Yamamoto-Hino \& Goto 2013). Coupling RNAi results with loss-of-function alleles is the best approach to uncovering gene function. However, many null and hypomorphic alleles result in lethality at various stages of development. The ability to dissect testes at larval stages of development can circumvent these problems if lethality occurs at late third-instar or pupal stages of development, thus allowing for analysis of gene function in early spermatogenesis. For example, our lab discovered a role for the RNA-binding protein Musashi (Msi) in germline stem cell maintenance by initially analysing third-instar and pupal gonads as msi mutant adult flies die soon after eclosion (Siddall et al. 2006).

Where lethality occurs during embryogenesis or where a gene is expressed in multiple cell types, FRT (flippase recognition target)-mediated recombination has been incredibly useful in elucidating gene function in specific cell populations in the testis. This technique allows for controlled induction of mitotic recombination events and is often performed by expressing the flippase (FLP) enzyme under the control of a heat-shock ( $h s$ ) promoter. A hs promoter enables the expression of high FLP levels at specific developmental time points. Activation of FLP allows for recombination between two homologous chromosome arms carrying flippase recognition target (FRT) sites in mitotically dividing cells, where one chromosome arm carries a GFP reporter under the control of a ubiquitous promoter, whereas the other carries a mutation in a gene of interest. Thus, homozygous mutant cells no longer expressing a marker such as GFP can be generated in an otherwise heterozygous background where the health of the organism is uncompromised (Xu \& Rubin 1993). Using this technique, our lab was able to delineate an intrinsic role for Msi in GSC maintenance, despite Msi being expressed in somatic cells of the niche in addition to the germline (Siddall et al. 2006). Figure 4A and B show examples of negatively marked clones in the testis. 

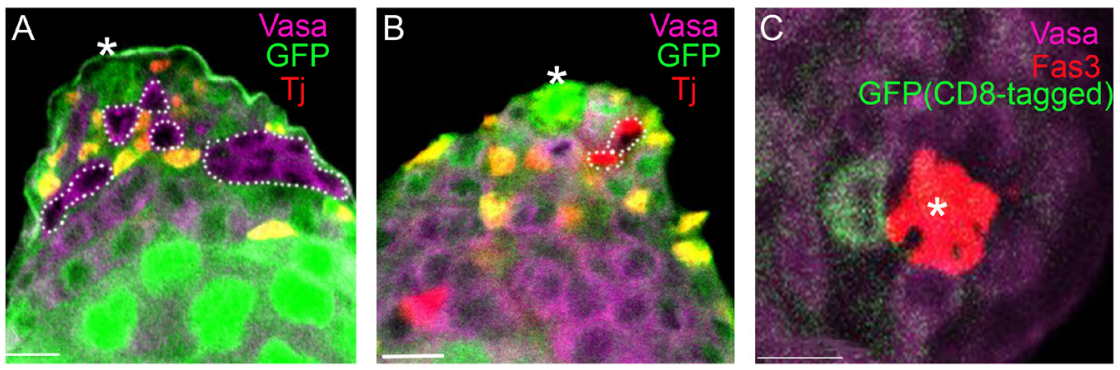

Figure 4 (A, B and C) Clonal analysis in adult testes using Flp-FRT-mediated recombination or MARCM analysis. The asterisk in each panel denotes the hub, scale bars represent $10 \mu \mathrm{m}$. (A) Flp-FRT-mediated recombination can be used to generate clonal cells (GFP-negative) and track clonal cells over time. The adult testis was dissected 7 days after heat shock treatment to generate wild-type clonal cells, and stained with antibodies against GFP (green), Vasa (germ cells, magenta) and Traffic Jam (cyst stem cells and differentiated cyst cells in testis apex (red). GFP-negative clonal germline stem cells and progeny can be visualized (outlined). (B) Flp-FRT-mediated recombination can be useful for generating clones in the somatic linage. The adult testis was dissected 3 days after heat shock treatment, and a clonal cyst progenitor cell labelled with Traffic Jam (red, outlined) and daughter can be observed. (C) MARCM allows for positively labelling cells and tracking cells over time. This adult testis dissected 2 days after heat shock treatment contains a GFP-positive germline stem cell clone (green, membrane-tagged GFP).

Development of the MARCM (Mosaic Analysis with a Repressible Cell Marker) technique allows for the positive labelling of a single cell or a group of cells related by lineage (Lee \& Luo 1999, del Valle Rodriguez et al. 2012). MARCM is also a FRT-based recombination technique, which utilises the addition of the GAL4-UAS system and the GAL80 represser to enable the expression of a marker such as GFP in homozygous mutant cells after inducing recombination by heat shock (Fig. 4C). In the heterozygous and homozygous wild-type tissue, a GAL80 transgene under the control of a ubiquitous promoter represses GAL4 activity and prevents the expression of the reporter GFP. FRT target recognition sites are located proximal to the mutation of interest in one chromosome arm and proximal to GAL80 in the homologous chromosome arm. Heat shockinduced mitotic recombination generates homozygous mutant clones that have lost the repressive GAL80 and are thus labelled by the expression of GFP. GFP can be visualized in all mutant clones if it is driven by a ubiquitous GAL4 driver or in only a subset of the mutant cells when using a specific GAL4 driver. This system has proved most useful to study the somatic cell lineage in the testis, with GFP-positive marked CySCs more readily visualized in whole testis tissue than negatively marked cells (Leatherman \& Dinardo 2008, Amoyel et al. 2014, 2016, Voog et al. 2014). MARCM also allows for the generation of clonal cells overexpressing a transgene because it combines Flp-FRT with the GAL4-UAS system. For example, Amoyel and coworkers recently deployed MARCM to show that Mitogen-Activated Protein Kinase (MAPK) is a major regulator of CySC competition for niche occupancy by creating positively marked clones specifically overexpressing MAPK (Amoyel et al. 2016). Thus, MARCM analysis allows for analysis of gene function in marked cells as well as lineage tracing.
Optimization of the lineage tracing system has occurred through the development of techniques such as G-Trace, which has further advanced the capabilities of Drosophila researchers (reviewed in Griffin et al. 2014). The G-Trace system combines the Gal4-UAS system with the Flp-based recombination system to enable a readout of GAL4 activity in real time, while at the same time allowing for permanent labelling of cells that express GAL4 or were derived from GAL4-expressing cells (Evans et al. 2009). In this system, a gene-specific GAL4 driver allows the expression of a reporter such as RFP (red fluorescent protein), thus reporting current gene expression. GAL4 simultaneously activates FLP expression also, which leads to the excision of a stop cassette and enables the expression of a second reporter, GFP, under the control of a ubiquitous promoter. GFP expression is permanent and inheritable and reports the history of expression of this gene. Therefore, G-TRACE provides both a spatial and a temporal readout of the expression of a gene of interest (reviewed in del Valle Rodriguez et al. 2012). G-Trace has been successfully utilised in the Drosophila testis to show a change of fate from hub cells to cyst cells upon loss of escargot (Voog et al. 2014). As other derivations of GAL4-UAS- and FRTbased technologies are enhanced, applications of newer technologies will be useful for future studies in Drosophila spermatogenesis.

Finally, a note should be made that FRT-based technologies are dependent upon recombination between homologous chromosomes; therefore, they exclude genes on the $X$ chromosome. Demarco and coworkers have nicely reviewed ways to circumvent this problem with the use of genomic rescue constructs or the actin-based flipout system (reviewed in Demarco et al. 2014), making the study of $X$ chromosome mutations in spermatogenesis easier to achieve. 


\section{Techniques for studying gene function at later stages in spermatogenesis}

So far, this review has focused on the range of genetic tools available for the study of processes in early spermatogenesis. The GAL4-UAS-based strategies rely on GAL4 lines driving the expression in cells in the apical section of the testis, with few GAL4 drivers able to successfully drive expression in spermatocytes (Bunt et al. 2012, White-Cooper 2012). In her review of spermatogenesis, White-Cooper notes that the likely reason some GAL4 lines do not work is related to the timing of GAL4 expression relative to the timing of shut-down of transcription that occurs in maturing primary spermatocytes, leading to the inability of GAL4 accumulation to high enough levels to drive the expression of a transgene. Therefore, expressing GAL4 in early primary spermatocytes with drivers such as bag-of marbles-GAL4 (bam-GAL4) can circumvent this problem, and some success using bam-GAL4 to drive RNAi in spermatocytes has been achieved (WhiteCooper 2012).

Phenotypic analysis of gene function at later stages of spermatogenesis relies heavily upon immunofluorescence or phase-contrast microscopy. Because spermatocytes are large, meiotic progression can easily be visualized in live testes by phase-contrast microscopy. In particular, haploid spermatids at a particular stage of development, referred to as the 'onion stage', can be easily observed using light microscopy techniques and can form the basis of genetic screens to identify factors involved in cytokinesis or chromosomal segregation (reviewed in Giansanti \& Fuller 2012). For example, by dissecting testes and simply gently squashing them under a coverslip and using phasecontrast microscopy to visualize the cells, we were able to identify meiotic defects in msi mutant animals (Fig. 5) (Siddall et al. 2006). Additionally, simple phase-contrast microscopy can be used to examine the number of early spermatocytes or early spermatids in cysts, allowing for the identification of mutations causing a reduction or increase in the number of spermatogonial divisions in the transit amplification stage (Monk et al. 2010). The simplicity of this technique in Drosophila makes it a useful tool for the identification of orthologous factors that may be involved in similar processes in mammalian systems. A comprehensive review on the recent discoveries of genes involved in cytokinesis and advantages of using Drosophila to study cytokinesis in male meiosis provides a nice summation of this topic (Giansanti \& Fuller 2012).

In Drosophila, the final stages of spermiogenesis, including chromatin re-organization, re-modelling of mitochondria and nuclei, formation of flagellar axonemes and acrosomes and polarisation of elongating cysts have been well described (Fabian \& Brill 2012). These post-meiotic events were originally believed to be driven by translation, as transcription of genes required for spermiogenesis were thought to occur in primary spermatocytes, with mRNAs stored in spermatids and translated during elongation (reviewed by Schafer et al. 1995). In contrast, transcription of genes required for mammalian spermiogenesis continues to occur postmeiotically (Schultz et al. 2003). By simple isolation of Drosophila spermatid bundles under a microscope on a siliconised slide, followed by quantitative RT-PCR, Barreau and coworkers were able to provide evidence of post-meiotic transcription in Drosophila spermatids (Barreau et al. 2008). In their paper, Barreau and coworkers nicely describe how cysts can be staged using various GFP or RFP-tagged reporter lines, such as Mst35Ba-GFP (protamine-GFP), H2A-mRFP1 (histoneRFP) and Tpl94D-GFP (transition protein-GFP). Using this technique, they were able to describe the initiation of transcription of comet and cup genes prior to the deposition of transition proteins, in mid-elongation spermatids expressing histone-RFP but not protamineGFP (Barreau et al. 2008), once again highlighting the advantages of using simple fluorescence, readily available reporter lines and microscopy in Drosophila to further our understanding of spermiogenesis.

\section{CRISPR/Cas9 genome editing to study all stages of spermatogenesis}

Genome editing technologies that take advantage of guiding different nuclease enzymes to selected target
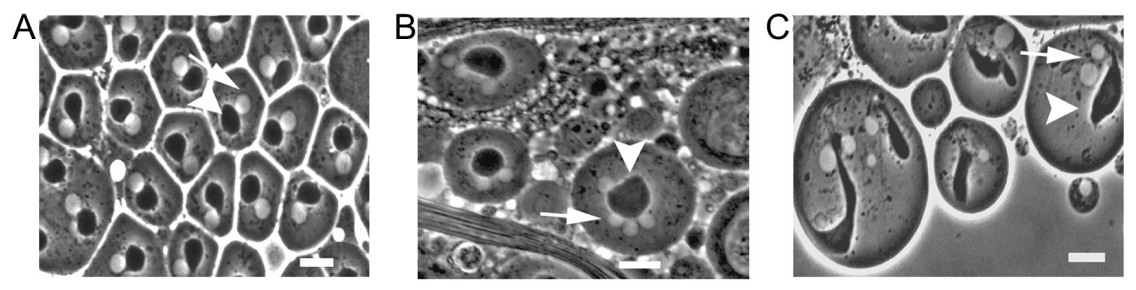

Figure 5 Phase-contrast imaging is a useful technique to study meiotic chromosome segregation and cytokinesis in the Drosophila testis. (A, B and C) Phase-contrast micrographs of postmeiotic spermatids in wild-type (A) and $\mathrm{msi}^{1}$ homozygous mutants (B and C). Arrowheads, mitochondrial derivatives; arrows, nuclei. (Scale bar: $10 \mu \mathrm{m}$.) (A) Onion-stage spermatids show one haploid nucleus and one mitochondrial derivative, each approximately the same diameter. (B) In $\mathrm{msi}^{1}$ mutants, two or four nuclei per mitochondrial derivative are often detected. (C) Nuclei in $\mathrm{msi}^{1}$ mutants are often different sizes (arrow). Adapted from Siddall et al. (2006). 
sites in the genome make it possible to engineer mutations in Drosophila and other organisms (Gaj et al. 2013, Mali et al. 2013, Beumer \& Carroll 2014). The most promising of these technologies is the CRISPR/ Cas9 (clustered regularly interspaced short palindromic repeats/CRISPR-associated) system. CRISPR/Cas-9 is a system that consists of two components, the Cas- 9 endonuclease that cleaves DNA, and the guide RNA that targets DNA sequences, thereby conferring target specificity. The RNA-guided DNA cleavage results in the formation of double-stranded breaks, which leads to small deletions and insertions and deletions (indels) at the target sites as a result of inefficient repair by nonhomologous end joining (NHEJ) (Bassett et al. 2013, Gratz et al. 2013). This technology enables researchers to generate mutations in any gene of interest. The study by $\mathrm{Wu}$ and coworkers, referred to at the beginning of this review, successfully used the CRISPR/Cas-9 technology in combination with other tools to uncover a conserved and specific requirement for the major spliceosome during the transition from spermatogonial proliferation to differentiation in the male testis (Wu et al. 2016). In their study, Wu and coworkers used Drosophila to evaluate the function of an SNP locus strongly associated with non-obstructive azoospermia (NOA) (Wu et al. 2016). Initially, using the GAL4-UAS system coupled with RNAi, they analysed the function of conserved genes identified within a genomic region closely associated with NOA. From this analysis, Wu and coworkers found that knockdown of U2A, the orthologue of hSNPRA1, resulted in male sterility in Drosophila. Using a range of genetic tools including CRISPR/Cas9 technology to create a mutant, construction of rescue constructs under the control of a heat-shock promoter allowing for conditional expression of a rescue transgene, cellspecific GAL4 lines for expression in the germline or soma and immunohistochemical analysis using cellspecific antibodies already described in this review. This study also revealed that $\mathrm{U} 2 \mathrm{~A}$, a component of the spliceosome, impairs spermatogonial differentiation and abolishes the maturation of germ cells into sperm (Wu et al. 2016). Interestingly, ubiquitous expression of hSNPRA1 in U2A knockdown flies both was able to restore fertility and rescue lethality of the mutant flies, showing functional conservation of the protein. Indeed, the authors reported that several missense mutations in human SNPRA1 that inhibit spliceosome assembly dominantly disrupt Drosophila spermatogonial differentiation (Wu et al. 2016). This study is a great example of how the use of CRISPR/Cas-9 can be coupled with other powerful genetic techniques in Drosophila to uncover the roles for previously unknown genes in human male fertility.

CRISPR/Cas-9 technology has recently been further enhanced in another study to uncover critical roles for long noncoding RNAs (IncRNAs) in Drosophila spermatogenesis. IncRNAs of greater than 200 nucleotides are a recently discovered class of cellular RNAs that play a role in the regulation of a wide range of cellular processes (for a detailed review on IncRNAs see Kung et al. 2013). Genome-wide transcriptome analyses have revealed that the largest repertoire of IncRNAs is expressed in the testis of Drosophila (Brown et al. 2014) and other species (Djebali et al. 2012, Nam \& Bartel 2012). Wen and coworkers developed the CRISPR/Cas-9 technology to conduct a large-scale gene knockout study of IncRNAs in the testis (Wen et al. 2016). By using a guide RNA, the Cas-9 mRNA and a homologous recombination donor plasmid to perform precisely targeted deletions of entire IncRNAs via homologous recombination, Wen and coworkers were able to rapidly delete 105 testis-specific Drosophila IncRNAs (Wen et al. 2016). Furthermore, their study showed that 33 of these deletion mutants exhibited reduced male fertility and that many IncRNAs are required for late spermatogenesis processes such as nuclear condensation and morphogenesis and spermatid individualization (Wen et al. 2016). This study clearly demonstrates how genome editing can rapidly uncover new aspects of regulation of spermatogenesis in Drosophila.

\section{Using Drosophila to study human male infertility}

Spermatogenesis is a highly conserved process from fly to human, and although some differences in the control of hormonal regulation and testicular structure have been described, many of the genes involved in Drosophila spermatogenesis are conserved in humans. A good example of this is Boule, where mutations in Drosophila boule cause sterility due to meiotic arrest, and mutations in a human orthologue, DAZ, result in azoospermia (Eberhart et al. 1996). Furthermore, insertion of a human paralog of DAZ (BOLL) transgene into Drosophila has been shown to rescue meiotic defects in boule mutant

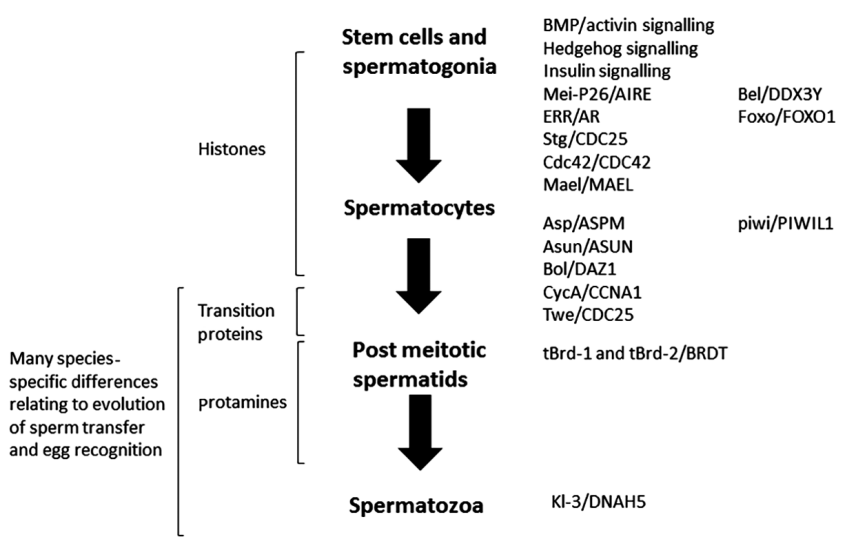

Figure 6 Schematic illustration of spermatogenesis with lists of conserved genes (Drosophila first followed by human gene) and gene pathways that have been associated with specific stages of germ cell development. Greater conservation of gene function is apparent in pre-meiotic and meiotic stages. 
flies (Xu \& Rubin 1993), suggesting that DAZ may encode a key switch that regulates the progression of germ cells in men. Some other examples of proteins that have been shown to have roles in both the fly germline and mammalian spermatogenesis include Drosophila Vasa and its murine orthologue $\mathrm{MVH}$, and Drosophila Piwi and its murine orthologue Miwi (Tanaka et al. 2000, Deng \& Lin 2002). A study by Bonilla and Xu used a genomic in silico approach to identify novel mammalian spermatogenic genes conserved from fly to human (Bonilla \& Xu 2008). Their approach to identify developmentally regulated testis-specific genes phylogenetically conserved across metazoans yielded 12 previously uncharacterized candidate fertility factors in mammals. This was amongst 58 identified genes, some of which had already been reported to be involved in spermatogenesis (Bonilla \& Xu 2008).

Many genes have been shown to have conserved roles in spermatogenesis between Drosophila and mammals (Fig. 6). This functional conservation is more often observed in pre-meiotic and meiotic stages and may relate to the greater interspecies morphological similarity observed between these cell types. Spermatozoa vary widely in morphology (even within mammalian groups), and the post-meiotic maturation processes also show some variation. That is not to say that processes such as histone replacement by protamines, and nuclear condensation probably utilise similar mechanisms in Drosophila and mammals. The role of spermatozoa is to fertilize eggs of the same species, and hence, in many cases, mechanisms have evolved to prevent interspecies fertilization. It is therefore not surprising that the greatest differences in male germ cell development would be observed in post-meiotic stages. Comparison of gene function between Drosophila species that have diverged by up to 50 million years of evolution does show some remarkable conservation of post-meiotic gene function. Examination of IncRNA function within Drosophila melanogaster spermatogenesis identified 33/105 IncRNAs that, when mutated via CRISPR, are necessary for late spermatogenesis. The IncRNAs that produce spermatogenic defects when mutated are much more highly conserved across the Drosophila species than other IncRNAs demonstrating that acquisition of a functional requirement during gametogenesis results in much slower evolutionary change (Wen et al. 2016).

This review has intended to draw attention to readers the vast range of genetic tools available in Drosophila, particularly for the study of spermatogenesis. With an estimated 2000 genes involved in human testicular development, germ cell differentiation, meiosis and successive stages of spermiogenesis (Bhasin et al. 2000), many of which are functionally conserved in Drosophila, flies allow a unique opportunity to study the potential role of these genes in fertility using a wide range of approaches.

\section{Declaration of interest}

The authors declare that there is no conflict of interest that could be perceived as prejudicing the impartiality of this review.

\section{Funding}

This work was funded by National Health and Medical research Council of Australia project grant APP1048110.

\section{References}

Amoyel M, Sanny J, Burel M \& Bach EA 2013 Hedgehog is required for CySC self-renewal but does not contribute to the GSC niche in the Drosophila testis. Development 140 56-65. (doi:10.1242/dev.086413)

Amoyel M, Anderson AM \& Bach EA 2014 JAK/STAT pathway dysregulation in tumors: a Drosophila perspective. Seminars in Cell and Developmental Biology 28 96-103. (doi:10.1016/j.semcdb.2014.03.023)

Amoyel M, Anderson J, Suisse A, Glasner J \& Bach EA 2016 Socs36E controls niche competition by repressing MAPK signaling in the Drosophila testis. PLoS Genetics 12 e1005815. (doi:10.1371/journal. pgen.1005815)

Barreau C, Benson E \& White-Cooper H 2008 Comet and cup genes in Drosophila spermatogenesis: the first demonstration of postmeiotic transcription. Biochemical Society Transactions 36 540-542. (doi:10.1042/BST0360540)

Bassett AR, Tibbit C, Ponting CP \& Liu JL 2013 Highly efficient targeted mutagenesis of Drosophila with the CRISPR/Cas9 system. Cell Reports $\mathbf{4}$ 220-228. (doi:10.1016/j.celrep.2013.06.020)

Beumer KJ \& Carroll D 2014 Targeted genome engineering techniques in Drosophila. Methods 68 29-37. (doi:10.1016/j.ymeth.2013.12.002)

Bhasin S, Mallidis C \& Ma K 2000 The genetic basis of infertility in men. Bailliere's Best Practice and Research: Clinical Endocrinology and Metabolism 14 363-388.

Bonilla E \& Xu EY 2008 Identification and characterization of novel mammalian spermatogenic genes conserved from fly to human. Molecular Human Reproduction 14 137-142. (doi:10.1093/molehr/ gan002)

Brand AH \& Perrimon N 1993 Targeted gene expression as a means of altering cell fates and generating dominant phenotypes. Development 118 401-415.

Brown JB, Boley N, Eisman R, May GE, Stoiber MH, Duff MO, Booth BW, Wen J, Park S, Suzuki AM et al. 2014 Diversity and dynamics of the Drosophila transcriptome. Nature $\mathbf{5 1 2}$ 393-399. (doi:10.1038/ nature12962)

Bunt SM \& Hime GR 2004 Ectopic activation of Dpp signalling in the male Drosophila germline inhibits germ cell differentiation. Genesis 39 84-93. (doi:10.1002/gene.20030)

Bunt SM, Monk AC, Siddall NA, Johnston NL \& Hime GR 2012 GAL4 enhancer traps that can be used to drive gene expression in developing Drosophila spermatocytes. Genesis 50 914-920. (doi:10.1002/ dvg.22341)

Caldwell JC, Joiner ML, Sivan-Loukianova E \& Eberl DF 2008 The role of the RING-finger protein Elfless in Drosophila spermatogenesis and apoptosis. Fly 2 269-279. (doi:10.4161/fly.7352)

Caussinus E, Kanca O \& Affolter M 2011 Fluorescent fusion protein knockout mediated by anti-GFP nanobody. Nature Structural and Molecular Biology 19 117-121. (doi:10.1038/nsmb.2180)

Chen D \& McKearin DM 2003 A discrete transcriptional silencer in the bam gene determines asymmetric division of the Drosophila germline stem cell. Development 130 1159-1170. (doi:10.1242/dev.00325)

de Cuevas M \& Matunis EL 2011 The stem cell niche: lessons from the Drosophila testis. Development 138 2861-2869. (doi:10.1242/ dev.056242)

del Valle Rodriguez A, Didiano D \& Desplan C 2012 Power tools for gene expression and clonal analysis in Drosophila. Nature Methods 9 47-55. (doi:10.1038/nmeth.1800) 
Demarco RS, Eikenes AH, Haglund K \& Jones DL 2014 Investigating spermatogenesis in Drosophila melanogaster. Methods 68 218-227. (doi:10.1016/j.ymeth.2014.04.020)

Deng W \& Lin H 2002 miwi, a murine homolog of piwi, encodes a cytoplasmic protein essential for spermatogenesis. Developmental Cell 2 819-830. (doi:10.1016/S1534-5807(02)00165-X)

Djebali S, Davis CA, Merkel A, Dobin A, Lassmann T, Mortazavi A, Tanzer A, Lagarde J, Lin W, Schlesinger F et al. 2012 Landscape of transcription in human cells. Nature 489 101-108. (doi:10.1038/nature11233)

Eberhart CG, Maines JZ \& Wasserman SA 1996 Meiotic cell cycle requirement for a fly homologue of human Deleted in Azoospermia. Nature 381 783-785. (doi:10.1038/381783a0)

Evans CJ, Olson JM, Ngo KT, Kim E, Lee NE, Kuoy E, Patananan AN, Sitz D, Tran P, Do MT et al. 2009 G-TRACE: rapid Gal4-based cell lineage analysis in Drosophila. Nature Methods 6 603-605. (doi:10.1038/ nmeth.1356)

Fabian L \& Brill JA 2012 Drosophila spermiogenesis: big things come from little packages. Spermatogenesis 2 197-212. (doi:10.4161/ spmg.21798)

Fabrizio JJ, Boyle M \& DiNardo S 2003 A somatic role for eyes absent (eya) and sine oculis (so) in Drosophila spermatocyte development. Developmental Biology $258 \quad 117-128 . \quad$ (doi:10.1016/S00121606(03)00127-1)

Flaherty MS, Salis P, Evans CJ, Ekas LA, Marouf A, Zavadil J, Banerjee U \& Bach EA 2010 Chinmo is a functional effector of the JAK/STAT pathway that regulates eye development, tumor formation, and stem cell selfrenewal in Drosophila. Developmental Cell 18 556-568. (doi:10.1016/j. devcel.2010.02.006)

Fuller MT 1993 Spermatogenesis. In The Development of Drosophila, pp 71-147. Ed A MaM-A Bate. Cold Spring Harbor, NY, USA: Cold Spring Harbor Laboratory Press.

Gaj T, Gersbach CA \& Barbas CF 3rd 2013 ZFN, TALEN, and CRISPR/Casbased methods for genome engineering. Trends in Biotechnology 31 397-405. (doi:10.1016/j.tibtech.2013.04.004)

Giansanti MG \& Fuller MT 2012 What Drosophila spermatocytes tell us about the mechanisms underlying cytokinesis. Cytoskeleton 69 869-881. (doi:10.1002/cm.21063)

Gnerer JP, Venken KJ \& Dierick HA 2015 Gene-specific cell labeling using MiMIC transposons. Nucleic Acids Research 43 e56. (doi:10.1093/nar/ gkv113)

Gonczy P, Viswanathan S \& DiNardo S 1992 Probing spermatogenesis in Drosophila with P-element enhancer detectors. Development 114 89-98.

Gratz SJ, Cummings AM, Nguyen JN, Hamm DC, Donohue LK, Harrison MM, Wildonger J \& O'Connor-Giles KM 2013 Genome engineering of Drosophila with the CRISPR RNA-guided Cas9 nuclease. Genetics 194 1029-1035. (doi:10.1534/genetics.113.152710)

Greenspan LJ, de Cuevas M \& Matunis E 2015 Genetics of gonadal stem cell renewal. Annual Review of Cell and Developmental Biology 31 291-315. (doi:10.1146/annurev-cellbio-100913-013344)

Griffin R, Binari R \& Perrimon N 2014 Genetic odyssey to generate marked clones in Drosophila mosaics. PNAS 111 4756-4763. (doi:10.1073/ pnas.1403218111)

Inaba M, Buszczak M \& Yamashita YM 2015 Nanotubes mediate nichestem-cell signalling in the Drosophila testis. Nature 523 329-332. (doi:10.1038/nature14602)

Kawase E, Wong MD, Ding BC \& Xie T 2004 Gbb/Bmp signaling is essential for maintaining germline stem cells and for repressing bam transcription in the Drosophila testis. Development 131 1365-1375. (doi:10.1242/ dev.01025)

Kelso RJ, Buszczak M, Quinones AT, Castiblanco C, Mazzalupo S \& Cooley L 2004 Flytrap, a database documenting a GFP protein-trap insertion screen in Drosophila melanogaster. Nucleic Acids Research 32 D418-D420. (doi:10.1093/nar/gkh014)

Kiger AA, Jones DL, Schulz C, Rogers MB \& Fuller MT 2001 Stem cell selfrenewal specified by JAK-STAT activation in response to a support cell cue. Science 294 2542-2545. (doi:10.1126/science.1066707)

Kung JT, Colognori D \& Lee JT 2013 Long noncoding RNAs: past, present, and future. Genetics 193 651-669. (doi:10.1534/genetics.112.146704)

Lai SL \& Lee T 2006 Genetic mosaic with dual binary transcriptional systems in Drosophila. Nature Neuroscience 9 703-709. (doi:10.1038/ $\mathrm{nn} 1681$ )
Lasko PF \& Ashburner M 1988 The product of the Drosophila gene vasa is very similar to eukaryotic initiation factor-4A. Nature 335 611-617. (doi:10.1038/335611a0)

Le Bras S \& Van Doren M 2006 Development of the male germline stem cell niche in Drosophila. Developmental Biology 294 92-103. (doi:10.1016/j.ydbio.2006.02.030)

Leatherman JL \& Dinardo S 2008 Zfh-1 controls somatic stem cell selfrenewal in the Drosophila testis and nonautonomously influences germline stem cell self-renewal. Cell Stem Cell 3 44-54. (doi:10.1016/j. stem.2008.05.001)

Leatherman JL \& Dinardo S 2010 Germline self-renewal requires cyst stem cells and stat regulates niche adhesion in Drosophila testes. Nature Cell Biology 12 806-811. (doi:10.1038/ncb2086)

Lee T \& Luo L 1999 Mosaic analysis with a repressible cell marker for studies of gene function in neuronal morphogenesis. Neuron 22 451-461. (doi:10.1016/S0896-6273(00)80701-1)

Li L \& Xie T 2005 Stem cell niche: structure and function. Annual Review of Cell and Developmental Biology 21 605-631. (doi:10.1146/annurev. cellbio.21.012704.131525)

Li MA, Alls JD, Avancini RM, Koo K \& Godt D 2003 The large Maf factor Traffic Jam controls gonad morphogenesis in Drosophila. Nature Cell Biology 5 994-1000. (doi:10.1038/ncb1058)

Lin H, Yue L \& Spradling AC 1994 The Drosophila fusome, a germlinespecific organelle, contains membrane skeletal proteins and functions in cyst formation. Development 120 947-956.

Mali P, Yang L, Esvelt KM, Aach J, Guell M, DiCarlo JE, Norville JE \& Church GM 2013 RNA-guided human genome engineering via Cas9. Science 339 823-826. (doi:10.1126/science.1232033)

McGuire SE, Mao Z \& Davis RL 2004 Spatiotemporal gene expression targeting with the TARGET and gene-switch systems in Drosophila. Science STKE 2004 pl6. (doi:10.1126/stke.2202004pl6)

McKearin D \& Ohlstein B 1995 A role for the Drosophila bag-of-marbles protein in the differentiation of cystoblasts from germline stem cells. Development 121 2937-2947.

Michel M, Raabe I, Kupinski AP, Perez-Palencia R \& Bokel C 2011 Local BMP receptor activation at adherens junctions in the Drosophila germline stem cell niche. Nature Communications 2 415. (doi:10.1038/ ncomms1426)

Michel M, Kupinski AP, Raabe I \& Bokel C 2012 Hh signalling is essential for somatic stem cell maintenance in the Drosophila testis niche. Development 139 2663-2669. (doi:10.1242/dev.075242)

Monk AC, Siddall NA, Volk T, Fraser B, Quinn LM, McLaughlin EA \& Hime GR $2010 \mathrm{HOW}$ is required for stem cell maintenance in the Drosophila testis and for the onset of transit-amplifying divisions. Cell Stem Cell 6 348-360. (doi:10.1016/j.stem.2010.02.016)

Nagarkar-Jaiswal S, DeLuca SZ, Lee PT, Lin WW, Pan H, Zuo Z, Lv J, Spradling AC \& Bellen HJ 2015a A genetic toolkit for tagging intronic MiMIC containing genes. Elife 4 e08469. (doi:10.7554/eLife.08469)

Nagarkar-Jaiswal S, Lee PT, Campbell ME, Chen K, Anguiano-Zarate S, Gutierrez MC, Busby T, Lin WW, He Y, Schulze KL et al. 2015b A library of MiMICs allows tagging of genes and reversible, spatial and temporal knockdown of proteins in Drosophila. Elife 4 e05338. (doi:10.7554/ eLife.05338)

Nam JW \& Bartel DP 2012 Long noncoding RNAs in C. elegans. Genome Research 22 2529-2540. (doi:10.1101/gr.140475.112)

Papagiannouli F \& Mechler BM 2009 Discs large regulates somatic cyst cell survival and expansion in Drosophila testis. Cell Research 19 1139-1149. (doi:10.1038/cr.2009.71)

Pfeiffer BD, Ngo TT, Hibbard KL, Murphy C, Jenett A, Truman JW \& Rubin GM 2010 Refinement of tools for targeted gene expression in Drosophila. Genetics 186 735-755. (doi:10.1534/genetics.110.119917)

Potter CJ \& Luo L 2011 Using the Q system in Drosophila melanogaster. Nature Protocols 6 1105-1120. (doi:10.1038/nprot.2011.347)

Schafer M, Nayernia K, Engel W \& Schafer U 1995 Translational control in spermatogenesis. Developmental Biology 172 344-352. (doi:10.1006/ dbio.1995.8049)

Schultz N, Hamra FK \& Garbers DL 2003 A multitude of genes expressed solely in meiotic or postmeiotic spermatogenic cells offers a myriad of contraceptive targets. PNAS $\mathbf{1 0 0}$ 12201-12206. (doi:10.1073/ pnas.1635054100)

Schulz C, Kiger AA, Tazuke SI, Yamashita YM, Pantalena-Filho LC, Jones DL, Wood CG \& Fuller MT 2004 A misexpression screen reveals 
effects of bag-of-marbles and TGF beta class signaling on the Drosophila male germ-line stem cell lineage. Genetics 167 707-723. (doi:10.1534/ genetics.103.023184)

Sheng XR \& Matunis E 2011 Live imaging of the Drosophila spermatogonial stem cell niche reveals novel mechanisms regulating germline stem cell output. Development 138 3367-3376. (doi:10.1242/dev.065797)

Siddall NA, McLaughlin EA, Marriner NL \& Hime GR 2006 The RNAbinding protein Musashi is required intrinsically to maintain stem cell identity. PNAS 103 8402-8407. (doi:10.1073/pnas.0600906103)

Sitaram P, Hainline SG \& Lee LA 2014 Cytological analysis of spermatogenesis: live and fixed preparations of Drosophila testes. Journal of Visualized Experiments 83 e51058. (doi:10.3791/51058)

Tanaka SS, Toyooka Y, Akasu R, Katoh-Fukui Y, Nakahara Y, Suzuki R, Yokoyama M \& Noce T 2000 The mouse homolog of Drosophila Vasa is required for the development of male germ cells. Genes and Development 14 841-853.

Terry NA, Tulina N, Matunis E \& DiNardo S 2006 Novel regulators revealed by profiling Drosophila testis stem cells within their niche. Developmental Biology 294 246-257. (doi:10.1016/j.ydbio.2006.02.048)

Tulina N \& Matunis E 2001 Control of stem cell self-renewal in Drosophila spermatogenesis by JAK-STAT signaling. Science $2942546-2549$. (doi:10.1126/science.1066700)

Van Doren M, Williamson AL \& Lehmann R 1998 Regulation of zygotic gene expression in Drosophila primordial germ cells. Current Biology $\mathbf{8}$ 243-246. (doi:10.1016/S0960-9822(98)70091-0)

Venken KJ, Schulze KL, Haelterman NA, Pan H, HeY, Evans-Holm M, Carlson JW, Levis RW, Spradling AC, Hoskins RA et al. 2011 MiMIC: a highly versatile transposon insertion resource for engineering Drosophila melanogaster genes. Nature Methods 8 737-743. (doi:10.1038/nmeth.1662)

Voog J, Sandall SL, Hime GR, Resende LP, Loza-Coll M, Aslanian A, Yates JR 3rd, Hunter T, Fuller MT \& Jones DL 2014 Escargot restricts niche cell to stem cell conversion in the Drosophila testis. Cell Reports 7 722-734. (doi:10.1016/j.celrep.2014.04.025)

Waclawska A \& Kurpisz M 2012 Key functional genes of spermatogenesis identified by microarray analysis. Systems Biology in Reproductive Medicine 58 229-235. (doi:10.3109/19396368.2012.693148)

Wen K, Yang L, Xiong T, Di C, Ma D, Wu M, Xue Z, Zhang X, Long L, Zhang W et al. 2016 Critical roles of long noncoding RNAs in Drosophila spermatogenesis. Genome Research 26 1233-1244. (doi:10.1101/ gr.199547.115)

White-Cooper H 2012 Tissue, cell type and stage-specific ectopic gene expression and RNAi induction in the Drosophila testis. Spermatogenesis 2 11-22. (doi:10.4161/spmg.19088)

Wu H, Sun L, Wen Y, Liu Y, Yu J, Mao F, Wang Y, Tong C, Guo X, Hu Z et al. 2016 Major spliceosome defects cause male infertility and are associated with nonobstructive azoospermia in humans. PNAS 113 4134-4139. (doi:10.1073/pnas.1513682113)

Xu T \& Rubin GM 1993 Analysis of genetic mosaics in developing and adult Drosophila tissues. Development 117 1223-1237.

Yamamoto-Hino M \& Goto S 2013 In vivo RNAi-based screens: studies in model organisms. Genes 4 646-665. (doi:10.3390/genes4040646)

Yamashita YM, Jones DL \& Fuller MT 2003 Orientation of asymmetric stem cell division by the APC tumor suppressor and centrosome. Science $\mathbf{3 0 1}$ 1547-1550. (doi:10.1126/science.1087795)

Yatsenko AN, Iwamori N, Iwamori T \& Matzuk MM 2010 The power of mouse genetics to study spermatogenesis. Journal of Andrology 31 34-44. (doi:10.2164/jandrol.109.008227)

Zhang Z, Pan C \& Zhao Y 2013 Hedgehog in the Drosophila testis niche: what does it do there? Protein and Cell 4 650-655. (doi:10.1007/s13238013-3040-y)

Zhao S, Chen D, Geng Q \& Wang Z 2013 The highly conserved LAMMER/CLK2 protein kinases prevent germ cell overproliferation in Drosophila. Developmental Biology 376 163-170. (doi:10.1016/j. ydbio.2013.01.023)

Zoller R \& Schulz C 2012 The Drosophila cyst stem cell lineage: partners behind the scenes? Spermatogenesis 2 145-157. (doi:10.4161/ spmg.21380)

Received 29 June 2016

First decision 20 September 2016

Revised manuscript received 15 December 2016

Accepted 10 January 2017 